\title{
Tratamiento del melanoma maligno en Colombia: resultados encuesta ACHO y Proyecto Grupo Colombo-Español del tratamiento multidisciplinario del melanoma
}

\footnotetext{
- Aylen Vanessa Ospina Serrano', Laura Bernal ${ }^{2}$

1 MD, Especialista en Medicina Interna y Oncología Clínica, Hospital Universitario Fundación Santa Fe de Bogotá. ICCAL. Coordinadora comité académico Oncología ACHO (Bogotá, Colombia). 2 MD, Medicina Interna, Especialista en Entrenamiento de Oncología Clínica. Instituto Nacional de Cancerología (Bogotá, Colombia).
}

\section{Introducción y antecedentes}

Teniendo en cuenta los datos demográficos, de acceso al sistema de salud y económicos de Colombia, se tiene una población estimada de 48,2 millones de habitantes, de los cuales el $76 \%$ reside en zona urbana. La economía se encuentra en desarrollo y actualmente hace parte del grupo de países de ingresos medios-altos, según el Banco Mundial'. Para 2015, alcanzó un índice de desarrollo humano de 0,727, categorizado como alto, y posicionándolo en el lugar 95 de 188 países en el mundo en este aspecto. Sin embargo, aún se encuentra por debajo del promedio de los países latinoamericanos, con un coeficiente de desigualdad del $23,7 \%$ y un índice de pobreza multidimensional del 7,6\% (un 5,7\% bajo la línea de pobreza y un 1,8\% en pobreza severa), con un $10,5 \%$ adicional de pobreza cercana a la multidimensional; el acceso a la educación es limitado, con una mediana de años escolares en el país de 72 .

Para el tratamiento de la patología oncológica, existen aproximadamente 200 especialistas en oncología y hematooncología; de los cuales, alrededor del 90\% son miembros activos de la Asociación Colombiana de Hematología y Oncología (ACHO).

Históricamente no se ha contado con un registro nacional de cáncer; se han venido estableciendo registros regionales (Barranquilla, Bucaramanga, Manizales, Pasto y Cali) y, recientemente, se han combinado los datos de los cuatro primeros registros ${ }^{3}$.
Revisando específicamente la información disponible en relación con el melanoma maligno, para el año 2012, agrupando los registros disponibles, se reportó una tasa de incidencia estandarizada de melanoma en Colombia de 1,7 por cada 100 mil personas-año y de mortalidad de $0,7^{3}$.

En el registro poblacional de Cali, se describe una tasa estandarizada de incidencia de 2,7 por cada 100 mil en el período comprendido entre 2008 y 2012 para los hombres, y de 2,5 para mujeres, con una tasa de mortalidad respectiva de 2,7 y $2,6^{4}$.

Datos del Instituto Nacional de Cancerología estiman que más del $50 \%$ de los pacientes se diagnosticaron en estadios avanzados y que las características clínicas son distintas a las de los países de alta prevalencia, con la principal diferencia de que el melanoma acral representa un $45 \%$ de los casos (contra un $5 \%$ al $10 \%$ descrito usualmente en la literatura mundial) $)^{5}$.

Un estudio local que incluyó 81 pacientes encontró una proporción del 19,8\% de lentiginosos acrales, con una frecuencia de BRAF mutado del $24,7 \%$ (menor a lo reportado usualmente) y un 70\% de tumores diagnosticados en estadio III o más ${ }^{6}$.

Durante los últimos años, en un intento de organizar la información generada con ánimo de fortalecer la evidencia en la cual se basa la modificación e implementación de políticas públicas, se ha propuesto la creación de un Observatorio Nacional de Cáncer (ONC) y se ha 
analizado la cuenta de alto costo. Cifras provenientes de esta última indican que para el período comprendido entre el 2 de enero de 2016 y el $1^{\circ}$ de enero de 2017 se registraron 3.326 casos de melanoma, representando el $1,7 \%$ de todos los casos nuevos de cáncer. La relación en la incidencia hombre:mujer consignada es de 1,6:1 y la edad media de presentación de 62,5 años, con un 91\% de los casos en mayores de 40 años.

De acuerdo con la tendencia mundial, se ha visto desde 2015 un aumento constante en el número de nuevos casos y en la tasa de mortalidad. El estadio al diagnóstico no se registró en más del $70 \%$ de los casos, y, para aquellos de los que se dispone de información, se diagnosticaron considerablemente menos en estadios tempranos en el régimen subsidiado que en el contributivo (figura 5). Fallecieron 279 pacientes por melanoma, lo que equivale a una mortalidad de 0,5 por 100 mil habitantes.

Con lo anterior expuesto, se evidencia que el melanoma en nuestro medio tiende a diagnosticarse en estadios avanzados, especialmente en estratos sociales bajos, donde la educación es limitada y existen demoras en el acceso a la atención médica de calidad. Teniendo en cuenta la desigualdad en la atención del cáncer en Colombia, nació desde la ACHO la iniciativa de formar un grupo académico de melanoma, con el apoyo del GEM (Grupo Español de Melanoma), como una herramienta para consolidar y compartir el conocimiento académico, en miras a estandarizar el manejo del melanoma en Colombia, y así poder brindar un tratamiento óptimo a los pacientes. Este grupo se conformó en septiembre de 2017 y cuenta actualmente con 22 miembros de las principales especialidades que manejan esta patología (oncología, hematooncología y radioterapia). El principal objetivo es generar y mantener la interacción entre los miembros de la ACHO y del GEM para realizar actividades académicas en conjunto, que incluyan cómites de tumores virtuales periódicos, reuniones de actualización, pasantías formativas e investigación colaborativa.

\section{Encuesta ACHO tratamiento del melanoma maligno en Colombia}

Como punto de partida, se aplicó en octubre de 2018 una encuesta para caracterizar el estado del melanoma en Colombia e identificar puntos importantes por trabajar, y en el primer semestre de 2018 se iniciaron las reuniones periódicas ACHO-GEM para planear actividades académicas. A continuación, se reportan los resultados de la encuesta:
Participaron 26 especialistas, en la figura 1 se muestra la distribución según el lugar de trabajo; en conjunto, se vieron representadas las principales ciudades del país.

Cada especialista estimó haber atendido en promedio a 16 pacientes en el último año, alcanzando en algunas instituciones más de 40 pacientes, con el mayor volumen en el Instituto Nacional de Cancerología (figura 2), seguido por Oncólogos del Occidente, Las Américas y Clínica Vida. El 62\% manifestó que su

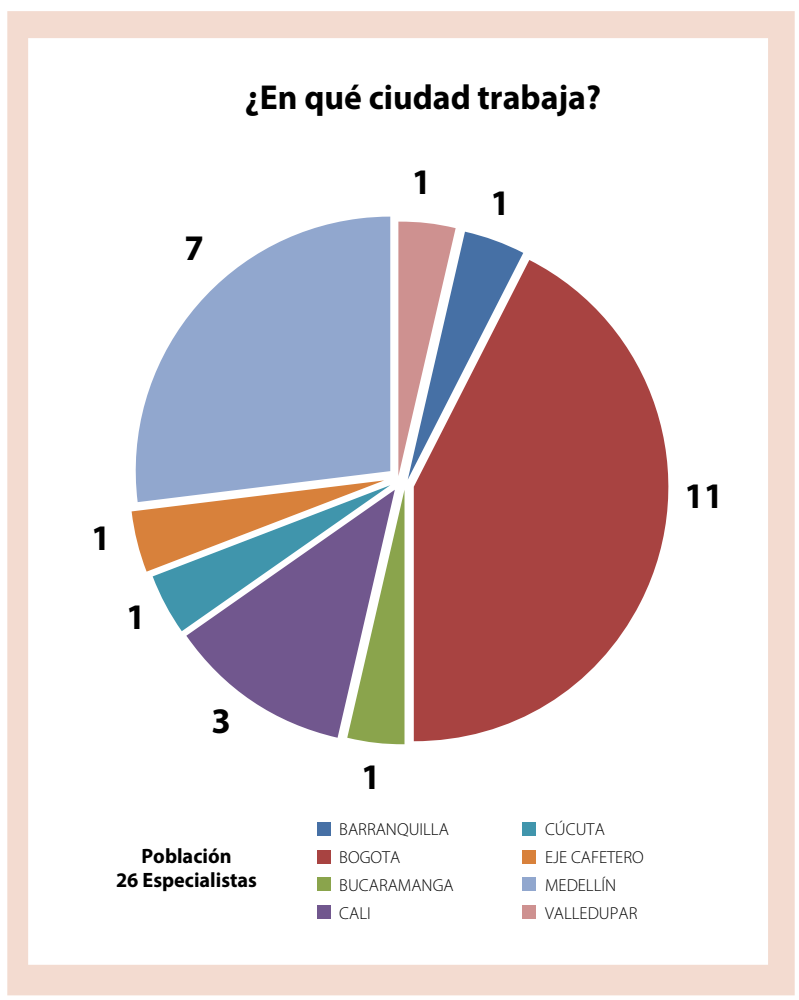

Figura 1

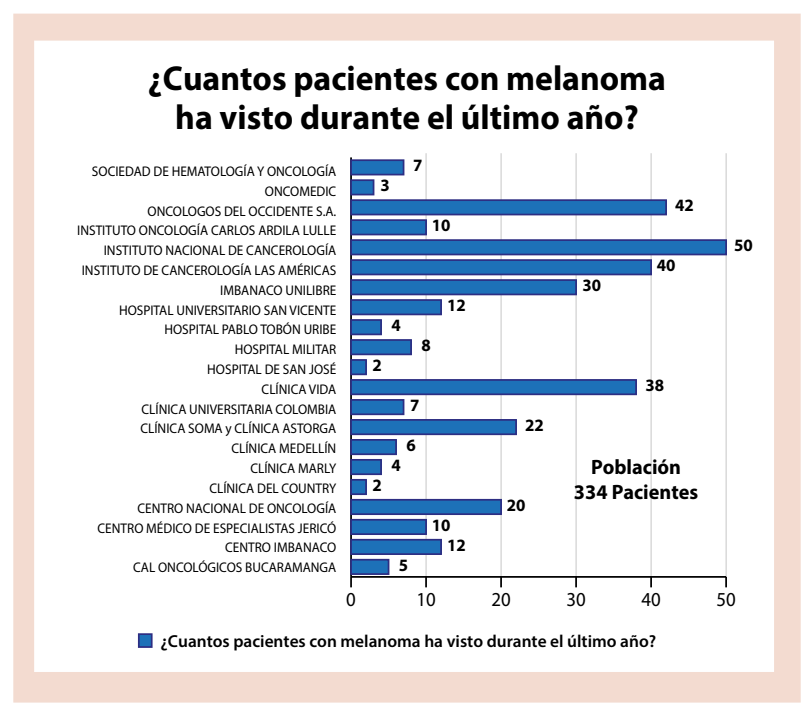

Figura 2 
institución no cuenta con una junta multidisciplinaria institucional de cáncer de piel para apoyar la toma de decisiones, el resto sí disponen de esta. La composición de dicha junta multidisciplinaria fue variable y está representada en la figura 3. Aproximadamente el 55\% de los pacientes consultan en un estadio metastásico de la enfermedad, proporción que fue similar para todas las instituciones, aunque en algunas alcanzó el $80 \%$ (figura 4).

\section{¿Quiénes componen la junta multidisciplinaria?}

13

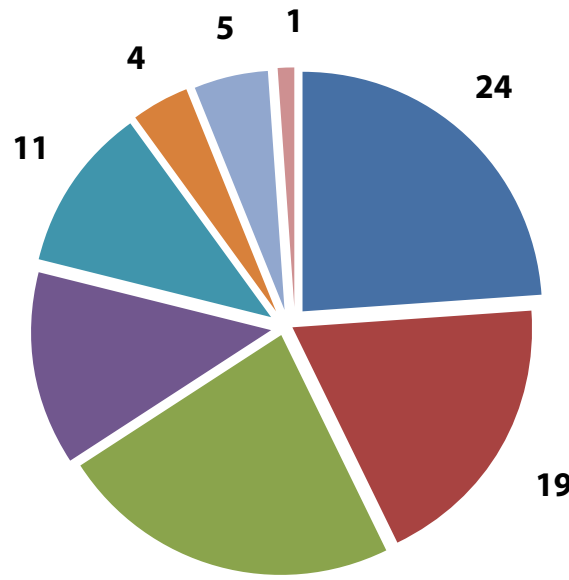

23

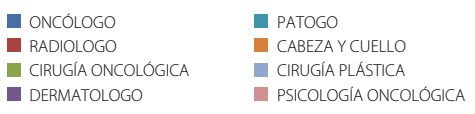

Figura 3

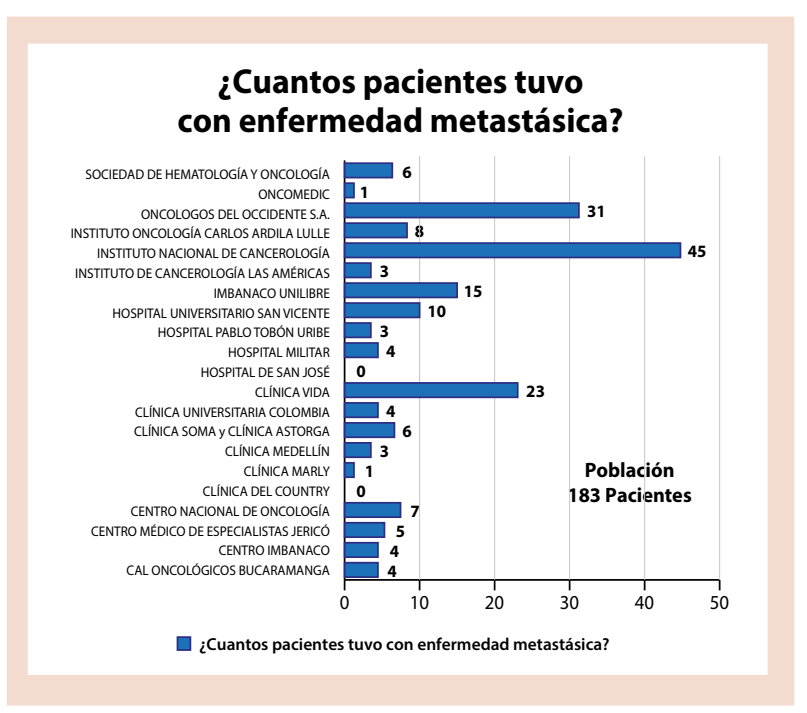

Figura 4
En el $90 \%$ de las instituciones, se realizó ganglio centinela de rutina al momento del manejo quirúrgico del primario, y en un $96 \%$ de los casos se ofreció manejo adyuvante. En el escenario adyuvante, fueron estadio III el $74 \%$ de los pacientes, el resto estadios II y IV resecado en proporciones iguales.

Como tratamiento adyuvante, el 76\% utilizó interferón- $\alpha-2 \mathrm{~B}$ a altas dosis, el $15 \%$ lo empleó a dosis intermedias. No se reportó uso de interferón a dosis bajas y el $9 \%$ manifestó manejar otros esquemas, como ipilimumab. Alrededor de la mitad refirieron solicitar el estado mutacional de BRAF en estadio IV y la otra mitad en estadios III y IV.

Los encuestados expresaron haber documentado mutaciones del BRAF en un $20 \%$ de los pacientes en estadio metastásico, y el $100 \%$ de los participantes afirmaron tener acceso al tratamiento dirigido con inhibidores de BRAF. No obstante, el $40 \%$ anotó haber tenido limitaciones de acceso a los fármacos anti-BRAF más nuevos de índole administrativa (autorizaciones por los aseguradores).

El 100\% manifestó tener acceso a inmunoterapia tanto con anti-PD1 (nivolumab, pembrolizumab) como a anti-CTLA4 (ipilimumab), aunque de nuevo el 36\% señaló algún tipo de limitación al momento de la autorización de estos medicamentos.

Se indagó acerca del criterio más importante para seleccionar el tratamiento de primera línea en pacientes con melanoma metastásico, y esto difirió según la biología del tumor. En pacientes con tumores BRAF mutados y aceptable estado funcional (ECOG menor de

¿Para seleccionar el tratamiento de primera línea a pacientes con melanoma metastásico BRAF mutado y buen estado funcional ECOG menor de 2, cuál es el criterio que considera más importante?

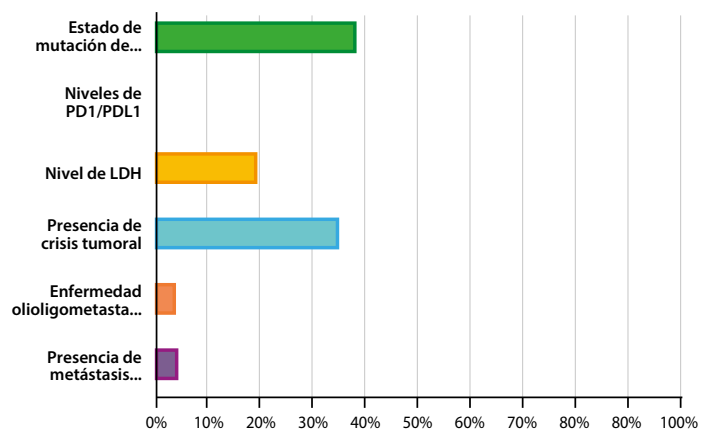

Figura 5 
¿Para seleccionar el tratamiento de primera línea a paciente con melanoma metastásico BRAF no mutado y buen estado funcional ECOG menor de 2, cuál es el criterio que considera más importante?

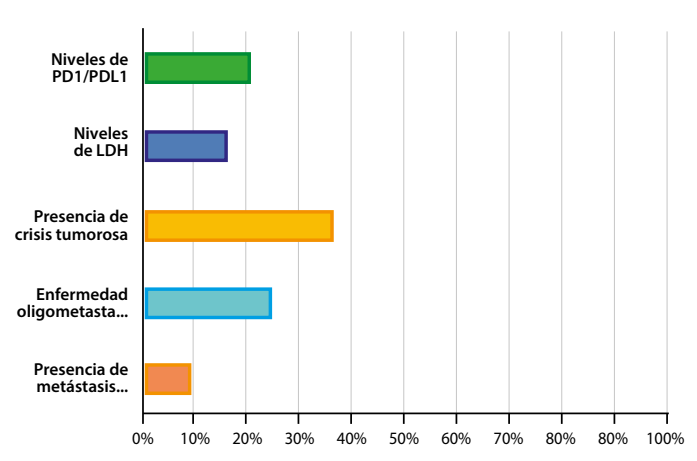

Figura 6

2), el criterio más favorecido fue el estado mutacional de BRAF en un $38 \%$ de los pacientes, seguido por la presencia de crisis tumoral en un 34\% y el nivel de LDH en el $20 \%$ (figuras 5 y 6 ).

En menor proporción, se dio importancia a la presencia de metástasis en el sistema nervioso central o al hecho de tratarse de una enfermedad oligometastásica.

Los encuestados no consideraron en este escenario relevantes los niveles de PD1/PD-L1. Por el contrario, para seleccionar el tratamiento de primera línea en pacientes con melanoma metastásico sin mutaciones del BRAF, con buen estado funcional (ECOG menor a 2), se consideró como criterio más significativo la presencia de crisis tumoral, seguido en orden por la presencia de pocos sitios de enfermedad (enfermedad oligometastásica), los niveles de LDH y, por último, la manifestación de metástasis en el sistema nervioso central.

El 32\% declaró hacer algún tipo de investigación básica en su hospital, y apenas el 9\% confirmó la elaboración de ensayos clínicos de melanoma en su centro en la actualidad, debido a regulaciones estatales para la realización de investigación clínica. A pesar de esto, alentadoramente el $92 \%$ de los encuestados expuso interés en participar en ensayos clínicos de melanoma.

\section{Discusión y conclusión}

La encuesta fue diligenciada voluntariamente por el $15 \%$ de los asociados, y permitió identificar que existen cuatro principales centros hospitalarios del país ubicados en las grandes ciudades que reciben el mayor flujo de pacientes con melanoma, y simultáneamente existen instituciones más pequeñas que reciben casos ocasionalmente.

La existencia de unos centros con mucho mayor volumen se da por referenciación asociada a disposiciones administrativas de los aseguradores y entes gubernamentales.

Aunque casi la totalidad de los participantes están interesados en participar en estudios clínicos, desafortunadamente la minoría no tiene actualmente la posibilidad de hacerlo en su institución debido a la dificultad de cumplir los requisitos gubernamentales para habilitar los centros de investigación. Por esto, de entrada una estrategia para optimizar el tratamiento del melanoma es fortalecer estos centros.

La atención del melanoma maligno en nuestro país es heterogénea, sin embargo, existe una proporción de pacientes adecuada para efectuar investigación clínica como una opción de tratamiento y de acceso a nuevos medicamentos. Por esto, es de gran relevancia aprovechar la interacción con el GEM e impulsar el Grupo Colombo-Español del tratamiento del melanoma para lograr estandarizar los manejos, aumentar el acceso a estudios clínicos, promover el diagnóstico temprano, el acceso oportuno a las terapias efectivas, fortalecer la educación médica continuada e incentivar la realización de juntas multidisciplinarias para definir en conjunto los tratamientos y conductas oncológicas.

Después de revisar los resultados de la encuesta, se ha planteado desde la ACHO la posibilidad de llevar a cabo un registro nacional de melanoma con el apoyo y orientación del GEM, para así ampliar y precisar la información acerca de la situación de esta patología en nuestro país, y poder abordar a las entidades gubernamentales y aseguradoras con datos sólidos y propuestas. Lo anterior con el objetivo de destacar la importancia de generar y fortalecer centros de referencia para esta patología, de manera que se garantice la mejor atención para los pacientes, entre otros, impulsando la creación de juntas multidisciplinarias en todas las instituciones con volumen significativo de pacientes, a fin de poder brindar una atención integral.

Además de enfatizar que debe favorecerse la posibilidad de los pacientes de participar en protocolos de investigación, siendo esta una herramienta terapéutica de gran valor e, incluso, una manera costo-efectiva para el sistema de salud de beneficiarse de las nuevas tecnologías y medicamentos en un país con las características socioeconómicas de Colombia. 


\section{Referencias}

1. http://databank.worldbank.org/

2. Human Development Report 2016 [internet]. Disponible en: http://hdr.undp.org/sites/all/themes/hdr_theme/country-notes/ COL.pdf

3. Información de cáncer en Colombia. Infocancer.co.

4. http://rpcc.univalle.edu.co/es/index.php
5. De Vries E. Melanomas in Colombia: a different reality. Rev Colomb Cancerol [internet]. 2013;17(3):91-2. Disponible en: http:// www.scielo.org.co/scielo.php?script=sci_arttext\&pid=S0123$90152013000300001 \& \operatorname{lng}=\mathrm{en}$

6. Carranza H, Archila P, Vargas C, Bernal L, et al. Genotipificación del melanoma en Colombia. Revista Colombiana de Hematología y Oncología. 2013;2(3). 